\title{
FRATURA DE CÔNDILO OCCIPITAL ASSOCIADA A FRATURA DE JEFFERSON E LESÃO DOS NERVOS CRANIANOS CAUDAIS
}

\section{Relato de caso}

\author{
Asdrubal Falavignaㄹ ${ }^{1}$ Fabrício Molon da Silva², Alessandra Souto Hennemann²
}

\begin{abstract}
RESUMO - A fratura de côndilo occipital (FCO) é patologia que pode passar despercebida à avaliação médica, não só por sua infrequência (1\%) como pela variedade de diagnóstico clínico e dificuldade na visualização dessa área ao exame radiológico de rotina. Essa fratura pode vir associada a lesões de nervos cranianos (31\%), sendo o hipoglosso o mais comumente envolvido (67\%). É rara sua associação com fratura de Jefferson. Relatamos o caso de uma paciente de 58 anos, que desenvolveu FCO, lesão de nervos cranianos caudais e fratura de Jefferson após uma queda. O tratamento preconizado foi tração cervical por seis semanas seguida pelo uso de halo-colete por três meses. Houve consolidação óssea e regressão da lesão nervosa. O objetivo deste relato é alertar para que, uma vez diagnosticada a FCO, está indicada investigação radiológica da transição crânio-cenvical pela possibilidade da presença de lesões simultâneas nervosas, de nervos cranianos e ósseas, como por exemplo a fratura de Jefferson. Apesar de suas associações serem raras, devem ter seu diagnóstico conhecido para poder ser manejadas adequadamente.
\end{abstract}

PALAVRAS-CHAVE: côndilo occipital, fratura de Jefferson, nervos cranianos.

\section{Occipital condyle fracture associated with Jefferson's fracture and injury of lower cranial nerves: case report}

ABSTRACT - Occipital condyle fracture (OCF) is rarely seen and can be missed during medical evaluation due to the variety of clinical presentations and the difficulty to be visualized radiographically. This fracture can be associated with cranial nerves injuries (31\%), being the hipoglossal nerve the most frequently involved (67\%). We report a 58 years old female patient who presented with OCF, injury of lower cranial nerves and Jefferson's fracture. The patient was treated with cervical traction for six weeks followed by halo immobilization for three months. There was bone consolidation recovery of the nervous injury after this period. This report emphazises the importance of investigating the skull-cenvical transition in all patients with cervical trauma. Although Jefferson's fracture is rarely associated with OCF, it should be remembered and treated appropriately when diagnosed.

KEY WORDS: occipital condyle fracture, Jefferson's fracture, cranial nerves palsy.

Descrita inicialmente por Sir Charles Bell ${ }^{1}$ em 1817 e posteriormente por Kissinger ${ }^{2}$ em 1900, a fratura de côndilo occipital (FCO) é pouco frequente e de difícil diagnóstico, pois o exame radiológico simples não evidencia a lesão e o quadro clínico émuito variável $^{3}$. Noble e Smoker ${ }^{3}$ constataram sua ocorrência em menos de $1 \%$ dos pacientes traumatizados, e associação com fraturas da coluna cervical em $20 \%$ dos casos. A FCO isolada não costuma vir acompa- nhada de distúrbio neurológico, sendo importante seu conhecimento pela possibilidade de evoluir para déficit neurológico de instalação tardia em decorrência da migração do fragmento ósseo fraturado ou de formação do calo ósseo ${ }^{3,4}$. Na presença de paralisia associada de nervos cranianos (NC), a mais frequente é a dos nervos caudais, podendo ter variadas combinações, desde paralisia isolada do hipoglosso até a de todos os nervos cranianos caudais ${ }^{5,6}$.

\footnotetext{
${ }_{1}^{1}$ Professor Adjunto da Disciplina de Neurologia da Universidade de Caxias do Sul, Caxias do Sul RS, Brasil, Pós-Graduando em Neurocirurgia pela Universidade Federal de São Paulo (UNIFESP); ${ }^{2}$ Acadêmico do 6 o Ano do Curso de Graduação em Medicina da Universidade de Caxias do Sul.
}

Recebido 28 Março 2002, recebido na forma final 29 Junho 2002. Aceito 14 Julho 2002.

Dr. Asdrubal Falavigna - Rua Coronel Camisão 241/301 - 95020-420 Caxias do Sul RS - Brasil. 
Tabela 1. Fratura de côndilo occipital associada com fratura de Jefferson e lesão de nervos cranianos caudais.

\begin{tabular}{|c|c|c|c|c|c|}
\hline Autor & Ano & $\begin{array}{c}\text { (FCO) } \\
\text { Número } \\
\text { de casos }\end{array}$ & $\begin{array}{c}\text { Fratura } \\
\text { Jefferson } \\
\text { associada }\end{array}$ & $\begin{array}{c}\text { Lesão } \\
\text { Associada } \\
\text { de PC }\end{array}$ & Recuperação da lesão do NC \\
\hline Bridgman Mcnab ${ }^{19}$ & 1992 & 1 & Sim & $\mathrm{X}, \mathrm{XI}, \mathrm{XII}$ & $\begin{array}{c}\text { Recuperação parcial do X e XII e } \\
\text { completa apenas do XI após um ano }\end{array}$ \\
\hline Stroobants et al. ${ }^{20}$ & 1994 & 1 & Sim & Não & - \\
\hline Noble et al. ${ }^{3}$ & 1996 & 15 & Sim (um caso) & Não & - \\
\hline Caso relatado & 2002 & 1 & Sim & $I X, X, X I I$ & Recuperação completa \\
\hline
\end{tabular}

$\mathrm{NC}$, nervos cranianos.

A fratura de Jefferson é uma fratura da primeira vértebra cervical (C1) do tipo explosão, em que observam três a quatro fraturas nos arcos anterior e posterior? Sua incidência é de 1-3\% das fraturas de coluna vertebral e de 4-15\% das da região cenvical, ocorrendo mais frequentemente na segunda década de vida e no sexo masculino $(2: 1)^{7-9}$. O mecanismo de lesão ocorre a partir de uma compressão axial aplicada ao vértice do crânio com a cabeça e o pescoço rígidos ${ }^{10}$.

Revisamos a literatura no período de 1817 a 2001 para averiguar os relatos de casos de FCO associados com lesão de NC caudais e fratura de Jefferson. Foram identificados 72 artigos, somando um total de 170 pacientes com FCO: 42 com lesão de NC associadas, três com fratura de Jefferson (Tabela 1), sendo descrito somente um caso com as três entidades.

\section{CASO}

Mulher de, 58 anos, casada, branca, previamente hígida, tropeçou e caiu de costas sofrendo trauma em regiões occipital e cervical superior. Levantou-se sem auxílio, referindo apenas desconforto na região occipital. Foi encaminhada ao serviço de pronto-socorro onde o exame neurológico mostrou, além da cervicalgia, lesão dos NC caudais: glossofaríngeo (IX NC), vago (X NC) e hipoglosso (XII NC), resultando em disfonia, disfagia e paresia da metade esquerda da língua, que progrediu para paralisia em uma hora (Fig 1). A investigação radiológica com raios-X simples, tomografia linear e tomografia computadorizada (TC) da transição crânio-cervical evidenciaram FCO esquerda (Fig 2) e fraturas múltiplas de Cl (Fig 3). Realizou-se tração cervical contínua com 4 kg durante seis semanas, com regressão dos sintomas e sinais dos NC inicialmente envolvidos. Transcorrido esse tempo e identificado radiologicamente o calo ósseo, indicou-se o uso de halo-colete, o qual foi mantido por três meses. Atualmente, após quatro anos do acidente, a paciente apresenta-se sem dor à movimentação da coluna cervical e com exame neurológico dos NC caudais dentro da normalidade. Como sequela observou-se discreta limitação do movimento de rotação, flexão e extensão da cabeça.

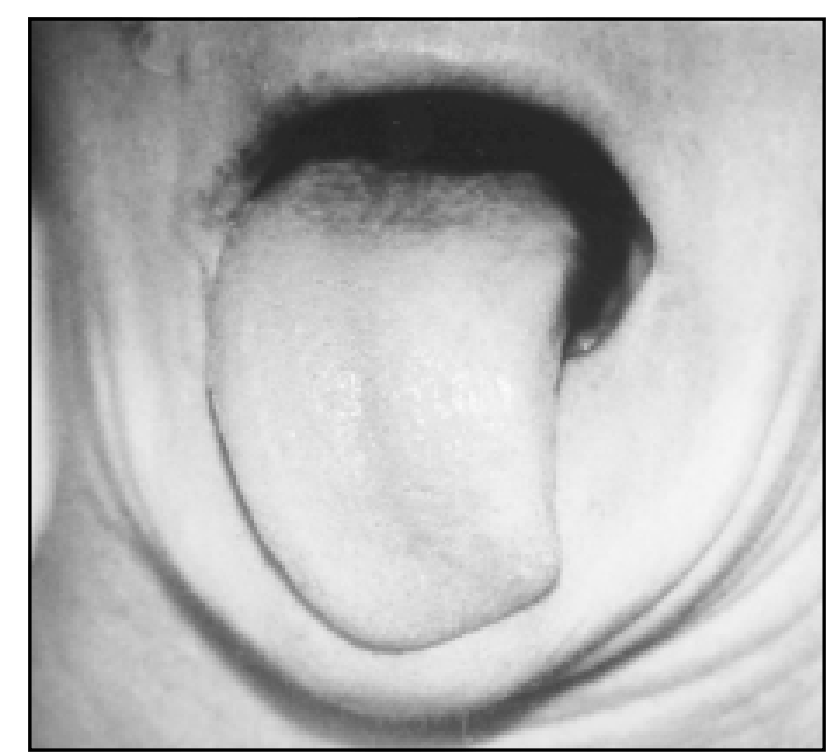

Fig 1. Protusão da língua com paresia do lado esquerdo.

\section{DISCUSSÃO}

A fratura-luxação da junção crânio-vertebral possui amplo espectro clínico, desde cervicalgia até lesões de nervos cranianos, tecido nervoso bulbar e medular, podendo evoluir para o óbito ${ }^{11}$. Embora considerada rara, a descrição de FCO aumentou significativamente nas duas últimas décadas, possivelmente em consequência do avanço dos equipamentos radiológicos ${ }^{11}$. Desde 1817 foram relatados 170 casos de FCO, sendo que $82 \%$ dos estudos foram publicados após o aparecimento da TC em 1983 ${ }^{11,12}$. O mecanismo provável da lesão é o movimento de hiperflexão e hiperextensão do pescoço associado à força vertical e de torção sobre a junção crâniocervical $^{6,11}$. A FCO usualmente ocorre isolada e bilateralmente, podendo haver associação com lesões na coluna cervical em $20 \%$ dos $\operatorname{casos}^{4,5,13}$. Miltner et al. ${ }^{14}$ diagnosticaram, na autópsia de 600 pacientes que faleceram por trauma resultante de acidente de 


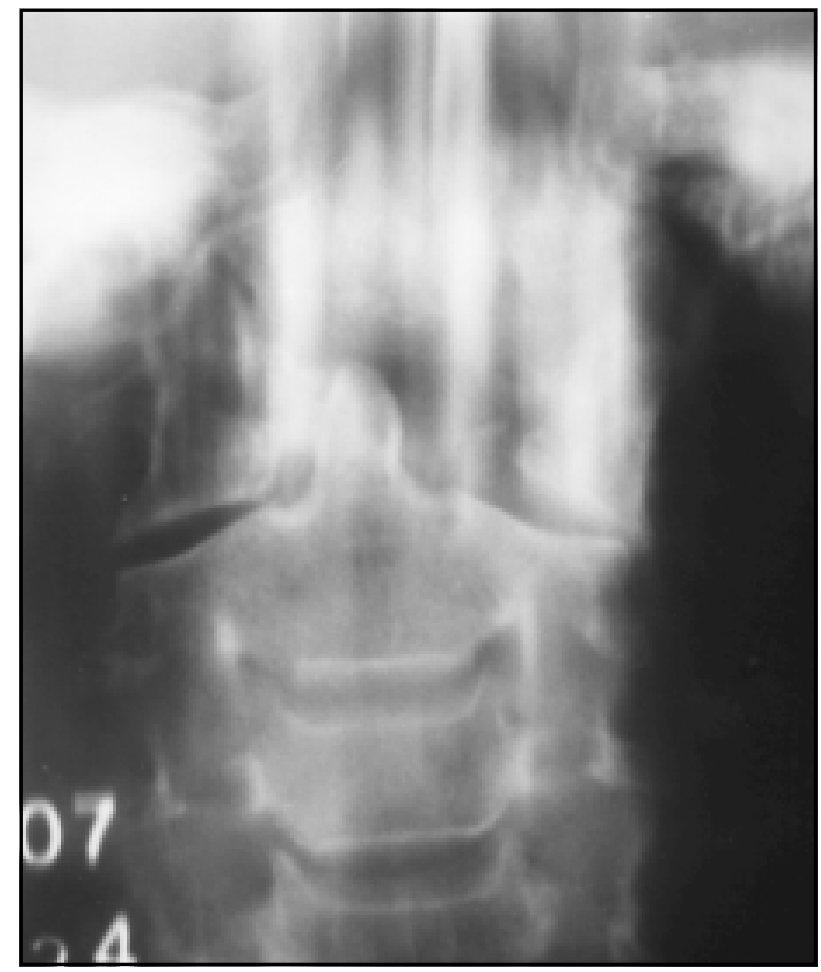

Fig 2. Tomografia linear (vista coronal) demonstrando fratura do côndilo occipital esquerdo.

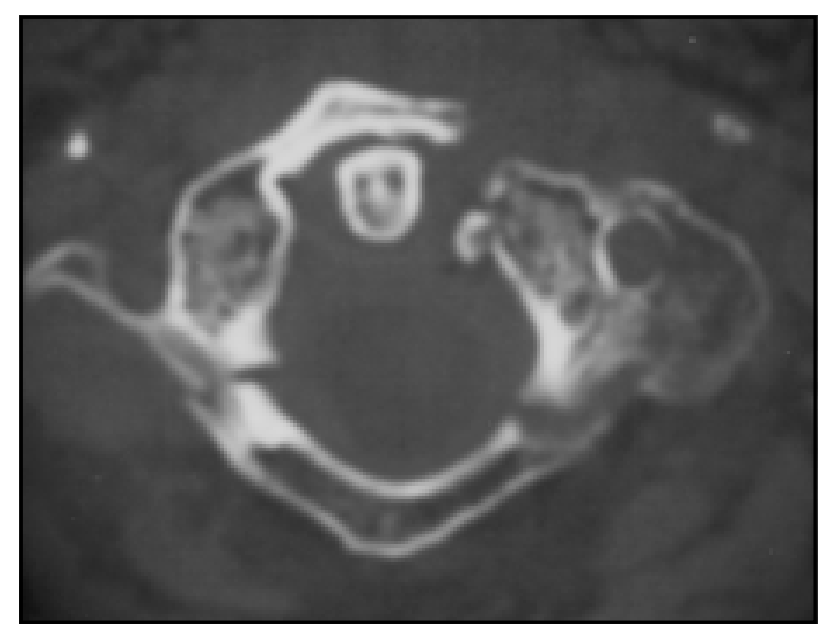

Fig 3. Corte axial de tomografia computadorizada onde se visualiza fraturas múltiplas dos arcos anterior e posterior de C1.

trânsito, 25 casos de FCO, com fratura bilateral de côndilo em 16 deles. As lesões concomitantes de NC caudais incidem em $31 \%$ dos casos de $\mathrm{FCO}^{5}$.

Os nervos mais acometidos são o hipoglosso, que atravessa o canal do côndilo occipital (canal do hipoglosso), e os NC IX, X e XI, que transitam pelo forâmen jugular, situado na proximidade do côndilo occipital $^{3}$. A distribuição de acometimento dos NC foi, nos 42 casos publicados, de 29 no XII NC, de 13 no XI NC, de 20 no X NC, de 16 no IX NC, de 10 no
VII NC, de 9 no VI NC e de 1 no V NC. A combinação de dois ou mais NC envolvidos foi encontrada em 26 casos, sendo a mais prevalente a do IX e XII NC, seguida pela XI e XII NC.

Em decorrência dos variados sintomas e sinais da apresentação clínica da FCO, alto índice de suspeita e exames de imagem são essenciais para seu diagnóstico ${ }^{5,6,11}$. O raio $X$ simples de crânio na incidência lateral ou AP não demonstra a FCO em razão da superposição de estruturas adjacentes da região mastoidéia e mandibular ${ }^{5}$. O diagnóstico definitivo é feito com TC convencional ou helicoidal, com cortes finos e reconstrução em plano sagital e coronal $^{13,15}$. Spencer et. al. ${ }^{13}$ afirmam que TC de escaneamento de alta resolução é o procedimento diagnóstico de escolha na FCO, por oferecer adequada visualização da base do crânio e não requerer movimento excessivo do paciente. A ressonância magnética (RM) de coluna cenvical pode complementar o estudo, por avaliar a integridade ligamentar e a compressão ou lesão medular ${ }^{16}$.

Em 1988, Anderson e Montesano ${ }^{15}$ propuseram uma classificação da FCO que correlaciona o mecanismo do trauma com a morfologia da fratura. Foram descritos três tipos de FCO: tipo 1, fratura de impacto com cominução e sem luxação dos fragmentos; tipo 2, fratura linear que envolve a base do crânio e passa através do côndilo; tipo 3, fratura em avulsão do côndilo occipital, com presença frequente de fragmento ósseo dentro do forâmen magno. Posteriormente, em 1997, Tuli et al. ${ }^{5}$ propuseram uma classificação mais funcional da FCO, enfatizando a mobilidade do complexo articular. Essa classificação é determinada pelo grau de lesão ligamentar refletido no deslocamento da FCO e pela determinação da instabilidade das articulações CO-C1-C2. Os tipos de FCO sugeridos pelos autores foram: tipo 1 , sem deslocamento; tipo 2A, com deslocamento e estabilidade do complexo articular CO-C1-C2; e tipo 2B, com instabilidade do complexo CO-C1-C2. Uma vez classificada a FCO, o tratamento sugerido será o do tipo 1, sem imobilização; no tipo 2A, colar cervical; e no tipo 2B, imobilização externa rígida (halo-colete) ou fixação cirúrgica. No caso relatado, a FCO foi do tipo 2 de Anderson e Montesso e do tipo 2B de Tuli.

Não há consenso na literatura sobre o tratamento da FCO. Bozboga et al. ${ }^{17}$ indicam, ocasionalmente, intervenção cirúrgica nas fraturas instáveis acompanhadas de complicações agudas do tipo compressão neurovascular. Outros autores estão de acordo com o tratamento conservador (halo-colete, tração cervical e colar cervical) nas fraturas instáveis acom- 
panhadas por lesões nervosas, observando grande percentagem de recuperação funcional ${ }^{4,11,18}$. Legros et al. ${ }^{4}$ observaram recuperação neurológica parcial nos 4 casos relatados. Em sua revisão literária, apenas quatro pacientes dos 18 casos se recuperaram completamente da lesão neurológica. Nos 170 casos descritos na literatura, para 30 foi prescrito repouso no leito; para os demais, o tratamento utilizado foi colar cervical (66 casos), halo-colete (8 casos), tração cenvical (2 casos) e cirurgia (6 casos). Não foi possível saber o tratamento realizado em 58 pacientes. $O$ único caso de associação de FCO com fratura de Jefferson e lesão de pares cranianos caudais foi manejado com uso de colar cervical ${ }^{19}$. Os dois casos de associação de FCO com fratura de Jefferson e sem lesão de NC foram tratados com colar cervical e o outro com halo-colete ${ }^{3,20}$. As FCO e a de Jefferson estáveis são tratadas usualmente com imobilização externa ${ }^{11}$.

No presente caso, não foi indicada cirurgia pelo risco de lesão adicional aos nervos cranianos caudais, que acontece, principalmente, no posicionamento do paciente em decúbito ventral, pois a monitorização neurofisiológica do IX e X NC é ineficaz e o controle clínico é prejudicado pela presença do tubo orotraqueal. Pelo fato de o mecanismo primário da fratura de Jefferson ser o de compressão axial, sua redução somente será obtida se aplicada uma força contrária de tração ${ }^{21}$. O uso imediato de halo-colete, além de não evitar a compressão axial da coluna cervical, não provê estabilização total da junção atlanto-occipital|21. Foi preconizada, no caso em estudo, tração cervical por seis semanas a fim de obter um calo ósseo, para posterior colocação de halo-colete.

\section{REFERÊNCIAS}

1. Bell C. Surgical observations. J Middelsex Hosp 1817;4:469-470.

2. Kissinger $P$. Luxations fraktur in atlanto occitpital gelenke. Zentrabl Chir 1900;37:933-934.

3. Noble E, Smoker W. The forgotten condyle: the appearence, morphology, and classification of occipital condyle fractures. Am J Neuroradiol 1996;17:507-513.

4. Legros $B$, Fournier $P$, Chiaroni $P$, Ritz $O$, Fusciard J. Basal fracture of theskull and lower $(I X, X, X I, X I I)$ cranial nerves palsy: four case reports including two fracture of the occipital condyle a literature review. J Trauma 2000;48:342-348.

5. Tuli S, Tator C, Fehlings M, Mackay M. Occipital condyle fractures. Neurosurgery 1997;41:368-377.

6. Gusmão S, Silveira R, Arantes A. Tratamento cirúrgico da fratura do côndilo occipital. Arq. Neuropsiquiatr 2001;59:134-137.

7. Scharen S, Jeannere T. A tlas fracture. Orthopade 1999;28:385-393.

8. Hadley M, Dickman C, Browner C, et al. A cutetraumatic atlas fractures: management and long term outcome. Neurosurgery 1988;23:31-35.

9. Lee C, Woodring J. Unstable Jefferson variant atlas fractures: an unrecognized cervical injury. AmJ Neuroradiol 1991;12:1105-1110.

10. Teo $\mathrm{E}, \mathrm{Ng} \mathrm{H}$. A nalytical static stress analysis of first cervical vertebra (atlas). Ann Acad Med Singapure 2000;29:503-509.

11. Leone A, Cerase A, Colosimoc C, Lauro L, Puca A, Marano P. Occipital condylar fractures: a review. Radiology 2000;216:635-644.

12. Peeters $F$, Verbeeten B. Evaluation of occipital condyle fracture and atlantic fracture, two uncommon complications of cranio-vertebral trauma. ROFO Fortschr Geb Roentgenstr Nuklearmed 1983;138:631-633.

13. Spencer J, Yeakley J, Kaufman H. Fracture of the occipital condyle Neurosurgery 1984;15:101-103.

14. Miltner E, Kallieris D, Schmidt G, Muller M. Injuries of the occipital condiles in fatal traffic accidents. Z Rechtsmed 1990; 103:523-528.

15. Anderson P, Montesano P. Morphology and treatment of occipital condyle fractures. Spine 1988;13:731-736.

16. Urculo E, Arrazola M, Arrazola M Jr, Riu I, Moyua A. Delayed glossopharyngeal and vagus nerveparalysisfollowing occipital condyle fracture. J N eurosurg 1996;84:522-525.

17. Bozboga $M$, Unal $F$, Hepgul $K$, et al. Fracture of the occipital condyle. case report. Spine 1992;17:1119-1121.

18. Hashimoto T, Watanabe O, Takase M, KonyamaJ, Kobota M. Collet-Sicard syndrome after minor head trauma. Neurosurgery 1988;23:367-370.

19. Bridgman S, Mcnab W. Traumatic occipital condyle fracture, multiple cranial nerves palsies and torticollis; a case report and review of the literature. Surg Neurol 1992;38:152-156.

20. Strobants J, Seynaeve P, Fidlers L, Klaes R, Brabants K, Van Hoye M. Occipital condyle fracture must be considered in the pediatric population: a case report. J Trauma 1994;36:440-441.

21. Koch R, Nickel V. The halo vest. an evaluation of motion and foces across the neck. Spine 1978; 3:103-107. 